\title{
A CONTROLLED TRIAL OF IVERMECTIN AND DIETHYLCARBAMAZINE IN LYMPHATIC FILARIASIS
}

\author{
Eric A. Ottesen, M.D., V. Vijayasekaran, M.D., V. Kumaraswami, M.D., S.V. Perumal Pillai, M.D., \\ A. Sadanandam, M.A., M.Phil., Sheila Frederick, B.S., R. Prabhakar, M.D., \\ and SRIRam P. Tripathy, M.D.
}

\begin{abstract}
Ivermectin is a new antifilarial drug that can be given in a single oral dose. To compare the efficacy and side effects of ivermectin with those of diethylcarbamazine, the standard antifilarial treatment, we conducted a randomized, double-blind trial in 40 South Indian men with lymphatic filariasis caused by Wuchereria bancrofti. Patients were randomly assigned to one of three treatments: a single low dose of ivermectin (mean [ $\pm S E$ ], $21.3 \pm 0.7 \mu \mathrm{g}$ per kilogram of body weight; $n=13$ ) followed by placebo for 12 days; a single high dose of ivermectin (mean, $126.2 \pm 3.7 \mu \mathrm{g}$ per kilogram; $n=13$ ) followed by placebo for 12 days; or diethylcarbamazine for 13 days $(6 \mathrm{mg}$ per kilogram per day for 12 days preceded by $3 \mathrm{mg}$ per kilogram for 1 day; $n=14$ ). Eleven patients were initially assigned to receive placebo and after five days were reassigned to one of the three treatment groups.
\end{abstract}

$\mathrm{I}^{\mathrm{V}}$ VERMECTIN is a semisynthetic macrolide antibiotic whose recent availability has entirely transformed strategies for the treatment and control of the filarial disease onchocerciasis, a major cause of blindness. Ivermectin destroys onchocerca microfilariae without triggering the violent side effects regularly induced by the older antifilarial drug diethylcarbamazine. Even a single oral dose of ivermectin is effective for at least 6 to 12 months. ${ }^{1-4} \mathrm{Lym}$ phatic filarial infections are by far the most prevalent: 90 million persons are infected worldwide with either Wuchereria bancrofti or Brugia malayi. ${ }^{5}$ Diethylcarbamazine, administered daily for one to two weeks, is the most widely used treatment, but it has proved difficult or impossible to use effectively in large, chemotherapy-based control programs. ${ }^{5}$ Thus, the possibility that ivermectin may have the same impact on the treatment and control of these infections that it has had on onchocerciasis merits urgent study and evaluation.

Early reports of open trials of ivermectin in patients infected with $W$. bancrofti have been encouraging: complete microfilaricidal activity has been seen after single oral doses that are comparable to ${ }^{6,7}$ or even much lower than $^{8}$ those used to treat onchocerciasis. The eradication of microfilariae was not permanent, however, since within six months after treatment, lowlevel microfilaremia recurred.$^{6-8}$ Furthermore, except for those taking the lowest dose of ivermectin $(25 \mu \mathrm{g}$

From the Laboratory of Parasitic Diseases, National Institute of Allergy and Infectious Diseases, National Institutes of Health, Bethesda, Md. (E.A.O.); the Clinical Pharmacology Unit, Madras Medical College and Government Hospital (V.V., S.V.P.P.), the Tuberculosis Research Centre (V.K., S.F., R.P.), and the Tamil Nadu Government Health Services (A.S.), all in Madras, India; and the Indian Council of Medical Research, New Delhi, India (S.P.T.). Address reprin requests to Dr. Ottesen at the Laboratory of Parasitic Diseases, National Institute of Allergy and Infectious Diseases, National Institutes of Health, Bldg. 4, Rm. 126, Bethesda, MD 20892.

Supported by the Indian Council of Medical Research, the National Institutes of Health, the Tropical Disease Research Programme (World Health Organization), and Merck Sharp \& Dohme Research Laboratories.
At day 12 there was complete clearance of microfilariae from the blood in all 26 men who took ivermectin and in 11 of the 14 men who took diethylcarbamazine. At six months the numbers of detectable microfilariae (as a percentage of the pretreatment values) were 18.3 percent after lowdose ivermectin and 19.5 percent after high-dose ivermectin, as compared with 6.0 percent after diethylcarbamazine $(P<0.05)$. The side effects were confined to the first five days and were similar in the three treatment groups.

We conclude thai in lymphatic filariasis, the clinical response to a single dose of ivermectin compares favorably with that after the standard 12-day course of diethylcarbamazine. Given the practical advantages of single-dose administration, ivermectin should become a useful medication for the control of bancroftian filariasis. (N Engl J Med 1990; 322:1113-7.)

per kilogram of body weight), most patients had side effects, such as fever, headache, lightheadedness, and myalgia, in proportion to the microfilarial loads present before treatment. Patients taking the lowest dose of ivermectin, however, had significantly fewer adverse reactions than those taking higher doses (50, 100 , or $200 \mu \mathrm{g}$ per kilogram), and the side effects in these patients were unrelated to the numbers of microfilariae present before treatment. ${ }^{8}$

Although diethylcarbamazine has been used to treat lymphatic filariasis for more than 40 years, no adequately detailed studies have been carried out to determine the rates of adverse reactions and the longterm efficacy of diethylcarbamazine in decreasing microfilaremia ${ }^{9}$; thus, a comparison of ivermectin and diethylcarbamazine treatment cannot readily be made from the information available. Furthermore, since the administration of a single oral dose of ivermectin has an enormous potential practical advantage over the currently recommended 12-day course of diethylcarbamazine, ${ }^{5}$ we performed a double-blind, placebocontrolled trial to compare both the efficacy and side effects of a standard 12-day course of diethylcarbamazine (6 mg per kilogram per day) and a single oral dose of ivermectin, either $120 \mu \mathrm{g}$ per kilogram (similar to the dose used for onchocerciasis) or $20 \mu \mathrm{g}$ per kilogram. The lower dose has been associated with fewer side effects than the higher dose but is equally efficacious. $^{8}$

\section{Methods}

\section{Patient Population}

Forty men from South India (19 to 45 years of age; median, 27.5) with asymptomatic $W$. bancrofti infection manifested solely as microfilaremia (range, 25 to 6000 microfilariae per milliliter; median, 901 ) were studied. The microfilaremia had been identified during a screening program conducted by the Public Health Department of Tamil Nadu State, India. Except for their filarial infections, the men were generally healthy. Each patient consented to participate in the ivermectin trial after the study protocol had been explained. The 
protocol was approved by the ethics committees of the Indian Council of Medical Research, the National Institutes of Health, Merck Sharp \& Dohme Research Laboratories, and the drug controller of India.

\section{Clinical Evaluation}

The patients were hospitalized in the Clinical Pharmacology Unit of the Government General Hospital in Madras for three days before receiving the study drug or placebo and for at least two weeks thereafter. Pretreatment evaluation included a detailed clinical history, physical examination, a complete blood count (hematocrit and total and differential white-cell counts), serum-chemistry studies (bilirubin, aspartate aminotransferase, alanine aminotransferase, alkaline phosphatase, and creatinine levels), urinalysis, chest roentgenography, and electrocardiography. After treatment, vital signs and 22 clinical variables were monitored with the use of a detailed checklist ${ }^{8}$ every four hours for two days and daily thereafter. The severity of each clinical finding was graded on a scale of 0 to 3 (none, mild, moderate, or severe). Follow-up examinations carried out in the outpatient clinic one, three, and six months after treatment included a clinical history, physical examination, a complete blood count, and serum-chemistry studies.

\section{Study Design}

After their pretreatment evaluation the study patients were randomly assigned to one of four treatment groups by a trial monitor who was not actively participating in the study. The study was double-blind: neither the patient nor the evaluating physician was aware of the medication being given.

On the first day patients received one of the following, given in two identical-appearing capsules: $1 \mathrm{mg}$ of ivermectin (mean $[ \pm \mathrm{SE}]$ dose, $21.3 \pm 0.7 \mu \mathrm{g}$ per kilogram), $6 \mathrm{mg}$ of ivermectin (mean dose, $126.2 \pm 3.7 \mu \mathrm{g}$ per kilogram), $150 \mathrm{mg}$ of diethylcarbamazine (approximately $3 \mathrm{mg}$ per kilogram, which is half the normal daily dose), or placebo. For the next 12 days patients in the diethylcarbamazine group were given $300 \mathrm{mg}$ of diethylcarbamazine each morning; those in the two ivermectin groups received similarappearing placebo capsules. Since the placebo group was included to control only for side effects (not for drug efficacy) and since essentially all side effects occur during the first five days, ${ }^{8}$ these patients received placebo capsules for five days and then were randomly reassigned to one of the three active-drug groups according to a protocol maintained by the trial monitor. Thus, although the participating physicians and the crossover patients knew that they were not being given a placebo during their second round of treatment, they did not know which of the active drugs they were taking. Although such knowledge could exaggerate the reported side effects, a quantitative comparison (see below) indicated no significant differences in adverse reactions between those receiving active drug initially and those receiving it after five days of placebo ( $P>0.05$ for all three drug regimens). Furthermore, of the 11 patients initially in the placebo group, 3 were subsequently assigned to the diethylcarbamazine group and 4 to each of the ivermectin groups; thus, any potential bias of the study design was spread evenly among the three drug regimens. A total of 13 patients

Table 1. Levels of Microfilariae in the Blood before Treatment, According to Study Group.

\begin{tabular}{|c|c|c|c|}
\hline \multirow[t]{3}{*}{ Study Group } & \multicolumn{3}{|c|}{ Microfilarial Density* } \\
\hline & GEOMETRIC MEAN & MEDIAN & RANGE \\
\hline & \multicolumn{3}{|c|}{ no. $/ \mathrm{ml}$ of blood } \\
\hline Ivermectin, $1 \mathrm{mg}(\mathrm{n}=13)$ & 722 & 1000 & $22-1775$ \\
\hline Ivermectin, $6 \mathrm{mg}(\mathrm{n}=13)$ & 481 & 970 & $25-1750$ \\
\hline Diethylcarbamazine $(n=14)$ & 651 & 813 & $83-6000$ \\
\hline Placebo $(n=11) \dagger$ & 571 & 813 & $83-1550$ \\
\hline
\end{tabular}

*The value for each patient was the mean of the three pretreatment measurements

†The patients in this group were reassigned to one of the three active-drug groups after five days; see Methods for study design. were enrolled in each of the two ivermectin treatment groups, and 14 were enrolled in the diethylcarbamazine group.

\section{Assessment of Microfilaremia}

The levels of microfilariae in the blood were assessed three times before treatment and 12 hours, 36 hours, 5 days, 12 days, 1 month, 3 months, and 6 months after treatment. Because of the nocturnal periodicity of the parasites, $1 \mathrm{ml}$ of heparin-treated blood was collected between 9:30 p.m. and 10 p.m. and filtered through a polycarbonate membrane with a pore size of $3 \mu \mathrm{m}$ (Nuclepore, Pleasanton, Calif.). The membrane was then placed on a microscope slide, stained wth Giemsa stain, and examined for trapped microfilariae. The results were expressed as the number of microfilariae per milliliter of blood or as a percentage of the mean number before treatment for each patient.

\section{Assessment of Side Effects}

For each patient a total adverse-reaction score was generated as described previously ${ }^{8}$ by summing the recorded scores for 22 clinical variables: fever, rigor, diaphoresis, headache, lethargy, weakness, lightheadedness, myalgia, arthralgia, rash, anorexia, nausea, vomiting, abdominal pain, diarrhea, pharyngitis, cough, chest pain, rales, wheeze, adenopathy, and scrotal tenderness. These signs and symptoms were monitored at all observation points during the first five days after treatment was begun and encompassed essentially all side effects associated with treatment except for postural hypotension, which was recorded separately. All these scores had returned to 0 by the fifth day of treatment. ${ }^{8}$ The adverse-reaction scores were used to compare the side effects of the various drug and placebo regimens and to assess the relations between side effects and microfilaremia levels.

\section{Statistical Analysis}

Student's t-test or linear regression analysis was used to evaluate the findings. For comparisons involving either microfilarial counts or total adverse-reaction scores, the values were logarithmically transformed before analysis. All $P$ values of less than 0.05 were considered to indicate statistical significance.

\section{Results}

\section{Efficacy}

Before treatment the levels of microfilariae in the blood were comparable in all four study groups (Table 1). A single oral dose of either $1 \mathrm{mg}$ (mean [ $\pm \mathrm{SE}$ ] dose, $21.3 \pm 0.7 \mu \mathrm{g}$ per kilogram) or $6 \mathrm{mg}$ (mean dose, $126.2 \pm 3.7 \mu \mathrm{g}$ per kilogram) of ivermectin effected nearly complete clearance of the microfilariae in all patients in 36 hours, and total clearance in 5 or 12 days (Fig. 1). Although rapid in both groups, the rate of clearance was significantly greater in patients receiving the higher, 6-mg, dose of ivermectin than in those receiving the l-mg dose, as seen by the difference in the microfilarial counts at the 12-hour assessment point $(\mathrm{P}<0.05)$.

In the group receiving diethylcarbamazine, daily administration of the drug also led to almost complete clearance of microfilariae from the blood in five days (Fig. 1), but the rate of clearance was significantly lower than that in either of the groups receiving ivermectin, as judged by the microfilarial counts in the three groups at 36 hours $(P<0.05$ for the comparisons between the diethylcarbamazine group and both ivermectin groups). Continued administration of diethylcarbamazine for the full 13-day course resulted in the eventual clearance of microfilariae in all but 3 of 14 patients, and even in these patients the numbers of 


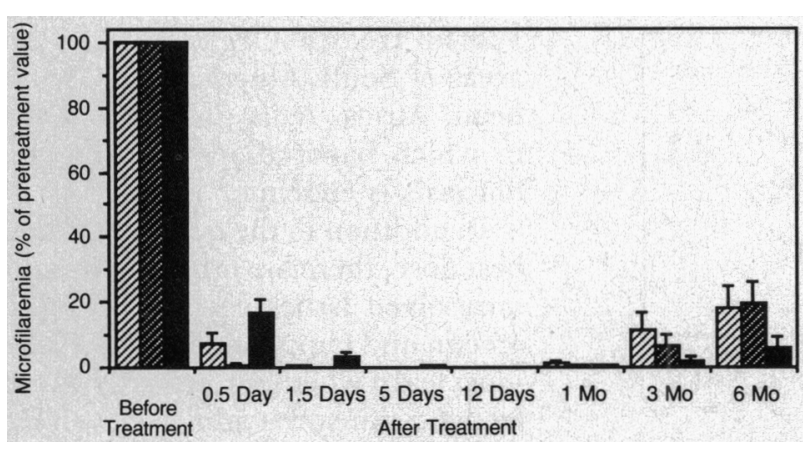

Figure 1. Clearance and Recurrence of Microfilariae in the Blood after either a 13-Day Course of Diethylcarbamazine (150 mg on Day 1 Followed by $300 \mathrm{mg}$ for 12 Days; Solid Bars) or a Single Oral Dose of $1 \mathrm{mg}$ (Mean $[ \pm \mathrm{SE}]$ Dose, $21.3 \pm 0.7 \mu \mathrm{g}$ per Kilogram; Lightly Hatched Bars) or $6 \mathrm{mg}$ (Mean Dose, 126.2 $\pm 3.7 \mu \mathrm{g}$ per Kilogram; Darkly Hatched Bars) of Ivermectin.

The geometric means (and standard errors) of the numbers of microfilariae in the blood, expressed as a percentage of the number present before treatment, are shown for each study group before treatment and at seven points after treatment $(0.5,1.5,5$, and 12 days and 1,3 , and 6 months).

microfilariae in the blood decreased to 0.1 to 0.3 percent of pretreatment values.

Within one month after treatment, microfilaremia began to recur at similar, very low levels in all three groups of patients. Three months after treatment, however, the levels of microfilariae in the blood were 11.2 percent and 6.5 percent (geometric means) of the pretreatment levels in patients treated with the $1-\mathrm{mg}$ and 6-mg doses of ivermectin, respectively ( $\mathrm{P}$ not significant), and at six months these levels had increased to 18.3 percent and 19.5 percent, respectively. For the patients treated with diethylcarbamazine, the corresponding levels of microfilariae in the blood at three and six months averaged 2.2 percent and 6.0 percent of the pretreatment levels (Fig. 1); these values were significantly lower $(\mathrm{P}<0.05)$ than those for both ivermectin-treated groups at six months and for the patients treated with $1 \mathrm{mg}$ at the three-month evaluation point.

\section{Side Effects Associated with Ivermectin and Diethylcarbamazine}

Although side effects were reported in all three treatment groups (as well as by the patients taking only placebo), the mean adverse-reaction scores of the diethylcarbamazine group (geometric mean, 43.5), the patients taking $1 \mathrm{mg}$ of ivermectin (geometric mean, 52.6), and those taking $6 \mathrm{mg}$ of ivermectin (geometric mean, 61.5) were significantly higher than those of the patients taking placebo alone (geometric mean, 15.7; $\mathrm{P}<0.02$ for all comparisons).

There were, however, no significant differences in the quantitative adverse-reaction scores among the three treatment groups, although there was a trend toward higher scores in the patients receiving highdose ivermectin $(6 \mathrm{mg})$. Qualitatively, the side effects were similar in the three groups: fever, headache, lethargy, and myalgia were the most common, beginning
12 to 24 hours after the medication was first given and subsiding in essentially all patients within 24 to 48 hours. Mild postural hypotension (i.e., a decrease in the mean arterial blood pressure in the standing position, accompanied by tachycardia ${ }^{8}$ ) occurred in one patient in each of the three groups 24 to 36 hours after treatment. All three had normal blood pressures at rest and were asymptomatic unless required to stand; their blood pressures returned to normal within 24 hours without specific treatment other than bed rest.

As in the previously reported open trial, ${ }^{8}$ the adverse-reaction scores of patients after treatment with the higher dose of ivermectin correlated significantly $(\mathrm{P}<0.01)$ with their pretreatment microfilaremia levels. Similarly, with respect to fever, the most objectively quantifiable adverse reaction, there were significant correlations with the numbers of microfilariae in the blood before treatment both for those taking ivermectin and for those receiving diethylcarbamazine (Fig. 2).

\section{Discussion}

Earlier open trials indicated that a single oral dose of ivermectin was a good but not perfect treatment for bancroftian filariasis. ${ }^{6-8}$ It caused rapid clearance of microfilariae from the blood, but some microfilaremia recurred three to six months after treatment, and transient side effects developed after drug administration. On the other hand, recurrence of microfilaremia is common after a 12-day course of diethylcarbamazine, the standard drug for the treatment of lymphatic filariasis. Furthermore, the acute side effects produced by diethylcarbamazine decrease the rate of compliance with the recommended 12-day course of treatment and have been responsible for the frequent failure of chemotherapy-based control programs.

To compare the relative merits of these two drugs in the treatment of bancroftian filariasis, we conducted a double-blind, placebo-controlled trial in which the optimal diethylcarbamazine regimen $(6 \mathrm{mg}$ per kilogram per day for 12 days, ${ }^{5}$ preceded by $3 \mathrm{mg}$ per kilogram for 1 day to minimize side effects) was tested against single oral doses of ivermectin, either $1 \mathrm{mg}$ (mean [ $\pm \mathrm{SE}$ ] dose, $21.3 \pm 0.7 \mu \mathrm{g}$ per kilogram) or $6 \mathrm{mg}$ (mean dose, 126.2 $\pm 3.7 \mu \mathrm{g}$ per kilogram). Follow-up lasted six months, a period in which any new infections should not be responsible for additional microfilariae in the blood.

The results of this comparison were clear. Ivermectin cleared the microfilariae from the blood more rapidly than did diethylcarbamazine. It was essentially equivalent to diethylcarbamazine in its ability to maintain this effect for up to three months after treatment, but by six months it did not sustain this decrease as well as diethylcarbamazine. Furthermore, the l-mg dose proved to be as effective as the 6-mg dose.

The two drugs produced essentially identical side effects, both qualitatively and quantitatively. As described previously for diethylcarbamazine $e^{9-13}$ and for the higher dose of ivermectin, ${ }^{8}$ the occurrence and 


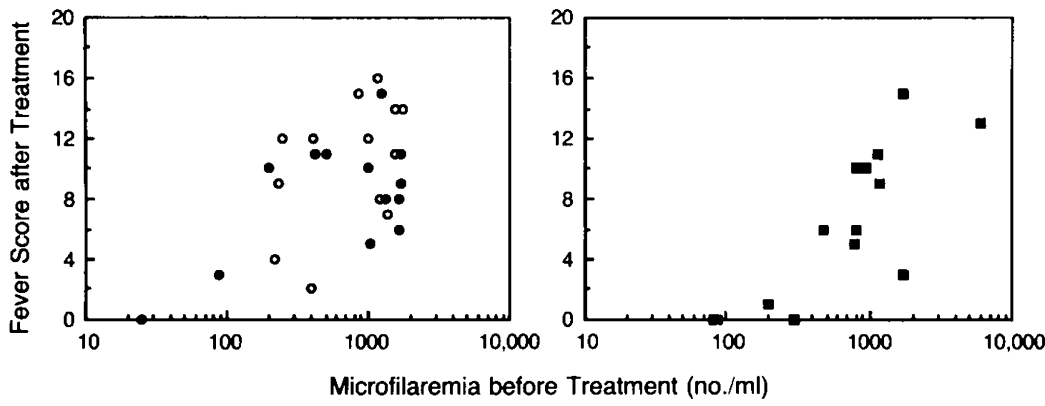

Figure 2. Fever Scores after Treatment as a Function of the Numbers of Microfilariae in the Blood before Treatment with Ivermectin (Left-Hand Panel) or Diethylcarbamazine (Right-Hand Panel).

The relation was statistically significant for the patients taking diethylcarbamazine (squares; $P<0.01$ ) and for those taking the 6-mg dose of ivermectin (solid circles; $P<0.05$ ). A similar trend was seen for the patients treated with the lower dose of ivermectin (1 $\mathrm{mg}$; open circles). The fever score is expressed in arbitrary units as defined previously. ${ }^{8}$

severity of these side effects generally correlated with the numbers of microfilariae in the blood before treatment. This observation implies that drug toxicity was not the direct cause of the side effects, but rather that the host inflammatory response to dying microfilariae was the chief determinant (by whatever mechanisms $\left.^{9,14-16}\right)$.

Given the effectiveness of ivermectin as a microfilaricide (in this study and in the earlier trials $s^{6-8}$ ), the general similarity of the clinical responses to ivermectin and diethylcarbamazine in the present study, the tremendous potential advantage of ivermectin's single-oral-dose mode of administration for use in largescale control programs, and the extensive safety experience with this drug (more than 300,000 people treated thus far in the onchocerciasis-control programs in Africa and Latin America ${ }^{17}$ ), ivermectin now appears to be ready for broader field testing to determine its acceptability in populations affected by $W$. bancrofti and to resolve such practical issues as the optimal dosing interval for maximal therapeutic effect. Indeed, only the question of whether $W$. bancrofti microfilariae in different parts of the world are equally susceptible to ivermectin must be resolved before such trials are undertaken.

A previous trial in Tahiti $^{7}$ (and less complete observations in Senegal ${ }^{6}$ ) indicated that a dose of $\geqslant 100 \mu \mathrm{g}$ of ivermectin per kilogram was more effective than one of $50 \mu \mathrm{g}$ per kilogram in decreasing the levels of microfilaremia and was not accompanied by more frequent side effects. Our own studies in South India, however, have indicated equally clearly that doses of 20 to $25 \mu \mathrm{g}$ of ivermectin per kilogram were just as effective as those up to $200 \mu \mathrm{g}$ per kilogram in reducing the degree of microfilaremia and, importantly, were associated with either significantly fewer side effects ${ }^{8}$ or a tendency toward fewer side effects (in the present study) than occurred with higher doses. The reasons for these observed differences in the efficacy and side effects of ivermectin, in the various areas in which $W$. bancrofti is endemic, are currently being explored in a series of dose-defining trials (coordinated by the Tropical Disease Research Programme of the
World Health Organization) in areas of South America, the Caribbean, Africa, Asia, and the Pacific in which bancroftian and brugian filariasis is endemic.

In addition to the question of the best dose, there are other important unresolved issues concerning ivermectin and lymphatic filariasis. We must learn whether ivermectin is effective against the adult (as well as the microfilarial) stage of the parasite because such knowledge will have a major influence on how the drug is used. In onchocerciasis, for example, since ivermectin has no activity against the adult stage of the parasite, it must be given repeatedly every 6 to 12 months. The primary goal of such treatment is to decrease the levels of microfilariae and, thereby, to interrupt transmission and diminish microfilaria-related pathology. If a similar strategy is adopted for bancroftian filariasis, current findings $\mathrm{s}^{6-8}$ suggest that single doses of ivermectin may have to be repeated every three to six months to achieve similar reductions in the numbers of microfilariae. In addition, ivermectin may be effective in killing the L3 and L4 larval stages of the lymphatic filarial parasites (as it is in the dog heartworm, Dirofilaria immitis, but not in onchocerca species ${ }^{16}$ ) and thus may have potential as a prophylactic agent against lymphatic filarial infection.

Although it will take time to resolve these questions, the results of our present study, a direct comparison of ivermectin and diethylcarbamazine in lymphatic filariasis, offer important encouragement by showing that a single low dose of ivermectin administered orally compares well with the standard 12-day course of diethylcarbamazine. It is appropriate, therefore, to hasten the initiation of the new trials necessary to address the remaining unanswered questions in order to determine with certainty whether ivermectin will have the same dramatic effect in controlling lymphatic filariasis worldwide that it has had in the control of onchocerciasis in Africa and Latin America.

We are indebted to Mr. D. Neu and the late Dr. M.A. Aziz of Merck Sharp \& Dohme Research Laboratories for their assistance with the trial design and for supplying the medication and placebos used in this study, and to Dr. Brian White-Guay of Merck Sharp \& Dohme Research Laboratories for performing a critical review of the study results and manuscript.

\section{REFERENCES}

1. Greene BM, Taylor HR, Cupp EW, et al. Comparison of ivermectin and diethylcarbamazine in the treatment of onchocerciasis. N Engl J Med 1985; 313:133-8

2. Awadzi K, Dadzie KY, Schulz-Key H, Gilles HM, Fulford AJ, Aziz MA The chemotherapy of onchocerciasis. XI. A double-blind comparative study of ivermectin, diethylcarbamazine and placebo in human onchocerciasis in northern Ghana. Ann Trop Med Parasitol 1986; 80:433-42.

3. Diallo S, Aziz MA, Lariviere M, et al. A double-blind comparison of the efficacy and safety of ivermectin and diethylcarbamazine in a placebo controlled study of Senegalese patients with onchocerciasis. Trans R Soc Trop Med Hyg 1986; 80:927-34. 
4. Newland HS, White AT, Greene BM, et al. Effect of single-dose ivermectin therapy on human Onchocerca volvulus infection with onchocercal ocular involvement. Br J Ophthalmol 1988; 72:561-9.

5. WHO Expert Committee on Filariasis. Lymphatic filariasis: fourth report. WHO Tech Rep Ser 1984; 702:3-112

6. Diallo S, Aziz MA, Ndir O, Badiane S, Bah IB, Gaye O. Dose-ranging study of ivermectin in treatment of filariasis due to Wuchereria bancrofti. Lancet 1987; 1:1030.

7. Roux J, Cartel J-L, Perolat P, et al. Étude de l'ivermectine pour le traitement de la filariose lymphatique due a Wuchereria bancrofii var. Pacifica en Polynesie Française. Rev Soc Pathol Exotique 1989; 82:72-81.

8. Kumaraswami V, Ottesen EA, Vijayasekaran V, et al. Ivermectin for the treatment of Wuchereria bancrofti filariasis: efficacy and adverse reactions. JAMA 1988; 259:3150-3

9. Ottesen EA. Description, mechanisms and control of reactions to treatment in the human filariases. Ciba Found Symp 1987; 127:265-83.

10. Sundaram RM, Koteswara Rao N, Krishna Rao C, Krishna Rao P, Rao CK. Studies on Bancroftian filariasis control with diethylcarbamazine. I. Frequency and nature of drug reactions. J Commun Dis 1974; 6:290300 .
11. Ottesen EA. Efficacy of diethylcarbamazine in eradicating infection with lymphatic-dwelling filariae in humans. Rev Infect Dis 1985; 7:341-56.

12. Sasa M, Oshima T, Sato K, et al. Studies of epidemiology and control of filariasis: observations on the carriers of Wuchereria bancrofti in the Amami Islands with special reference to the effects and side-reactions of diethylcarbamazine. Jpn J Exp Med 1963; 33:213-43.

13. Ciferri FE, Kessel JF. Relation of age, sex, and microfilaria density to treatment of sub-periodic filariasis with diethylcarbamazine. Am J Trop Med Hyg 1967; 16:321-8.

14. Piessens WF, Beldekas M. Diethylcarbamazine enhances antibody-mediated cellular adherence to Brugia malayi microfilariae. Nature 1979;282:8457 .

15. Cesbron J-Y Capron A, Vargaftig BB, et al. Platelets mediate the action of diethylcarbamazine on microfilariae. Nature 1987; 325:533-6.

16. Campbell WC, ed. Ivermectin and abamectin. New York: Springer-Verlag, 1989:363.

17. World Health Organization. Report of a meeting of the TDR/OCP/OCT subcommittee for monitoring of community trials of ivermectin. Geneva: World Health Organization, 1989:16. (TDR/OCP/OCT/Ivermectin/ 89.3.)

\title{
LASTING REMISSIONS IN HAIRY-CELL LEUKEMIA INDUCED BY A SINGLE INFUSION OF 2-CHLORODEOXYADENOSINE
}

\author{
Lawrence D. Piro, M.D., Garlos J. Carrera, M.D., Dennis A. Carson, M.D., \\ and Ernest Beutler, M.D.
}

\begin{abstract}
Chlorodeoxyadenosine is a simple purine nucleoside that has previously been shown to be effective in the treatment of low-grade malignant disorders of lymphoid tissue, including chronic lymphocytic leukemia and non-Hodgkin's lymphoma. Because of these encouraging results, we treated 12 patients with another lowgrade B-cell neoplasm, hairy-cell leukemia. The patients received 2-chlorodeoxyadenosine $(0.1 \mathrm{mg}$ per kilogram of body weight per day) by continuous infusion for seven days.

All the patients responded to treatment. Eleven had complete remissions characterized by the normalization of peripheral blood and bone marrow and disappearance of
\end{abstract}

GIRST described by Bouroncle et al. in 1958 as I leukemic reticuloendotheliosis, 'hairy-cell leukemia is now recognized to be a low-grade $\mathrm{B}$-cell neoplasm. ${ }^{2}$ If untreated, this disorder follows a chronic, inexorable course, usually characterized by progressive pancytopenia.

Because of our success in the treatment of another B-cell neoplasm, chronic lymphocytic leukemia, with the deoxyadenosine analogue 2-chlorodeoxyadenosine ${ }^{3}$ we have now undertaken a trial of this agent in hairy-cell leukemia. An adenosine deaminase-resistant purine analogue, 2-chlorodeoxyadenosine selectively accumulates as the $5^{\prime}$-triphosphate derivative in cells rich in deoxycytidine kinase. The cytotoxic activity of 2-chlorodeoxyadenosine is independent of cell division. ${ }^{4}$ Presumably because of its high cellular specificity, 2-chlorodeoxyadenosine has previously

From the Department of Molecular and Experimental Medicine, Research Institute of Scripps Clinic, Scripps Clinic and Research Foundation, La Jolla, Calif. Address reprint requests to Dr. Piro at 10666 N. Torrey Pines Rd., La Jolla CA 92037.

Supported by grants (FD-R-000280, GM23200, and MO1 RR00833) from the National Institutes of Health, a Clinical Investigator Award (CA01 100), and the Alec Tapper Memorial Cancer Fund. tumor masses. The longest remission has been 3.8 years. None of the patients have relapsed, and the median duration of remission has been 15.5 months. No serious toxic reactions occurred as a result of 2-chlorodeoxyadenosine therapy.

These results suggest that 2-chlorodeoxyadenosine may be the most effective therapy available for hairy-cell leukemia. The administration of 2-chlorodeoxyadenosine resulted in a higher rate of complete remission than is observed with interferon alfa, and it required no maintenance therapy. Its toxicity may be lower than that of deoxycoformycin, and the responses were achieved with single courses of treatment. (N Engl J Med 1990; 322:1117-21.)

been found to be remarkably devoid of the toxic side effects usually associated with chemotherapeutic agents against cancer. Unlike deoxycoformycin, which has also been studied recently in the treatment of hairy-cell leukemia, 2-chlorodeoxyadenosine is not an adenosine deaminase inhibitor. Since both drugs increase the levels of deoxynucleotides, there are some similarities in their spectrums of activity.

We report the results of 2 -chlorodeoxyadenosine therapy in 12 patients with this relatively rare disorder. Our findings demonstrate that long-lasting remissions are induced with a single course of treatment without any serious side effects.

\section{Methods}

\section{Eligibllity}

To be eligible, patients required an unequivocal diagnosis of hairy-cell leukemia based on marrow-aspiration and biopsy results. Evidence of active disease, such as neutropenia, thrombocytopenia, anemia, symptomatic adenopathy, or repeated infections, was a prerequisite for entry into the study, but previous treatment was not. There were no age restrictions. Patients were excluded if they had active systemic infection or abnormal renal or hepatic function at the time of treatment, or if they had received any treatment, including glucocorticoids, within three months be- 